\title{
Nonlinear evolution problems with singular coefficients in the lower order terms
}

\author{
Fernando Farroni@, Luigi Greco®, Gioconda Moscariello@ and \\ Gabriella Zecca®
}

\begin{abstract}
We consider a Cauchy-Dirichlet problem for a quasilinear second order parabolic equation with lower order term driven by a singular coefficient. We establish an existence result to such a problem and we describe the time behavior of the solution in the case of the infinite-time horizon.
\end{abstract}

Mathematics Subject Classification. 35K55, 35K61.

Keywords. Parabolic equations, Time behavior.

\section{Contents}

1. Introduction 2

2. Preliminary results 4

2.1. Basic notation 4

2.2. Function spaces 4

$\begin{array}{ll}\text { 2.3. Gronwall type results } & 7\end{array}$

3. Weak type and a priori estimates for an auxiliary problem 8

4. Parabolic equations with bounded coefficients 12

5. Proof of the existence result via approximation scheme 15

6. Asymptotic behavior 18

Acknowledgements $\quad 22$

$\begin{array}{ll}\text { References } & 23\end{array}$

The authors are members of Gruppo Nazionale per l'Analisi Matematica, la Probabilità e le loro Applicazioni (GNAMPA) of INdAM. The research of G.M. has been partially supported by the National Research Project PRIN "Gradient flows, Optimal Transport and Metric Measure Structures", code 2017TEXA3H. 


\section{Introduction}

The aim of this paper is to study the following Cauchy-Dirichlet problem

$$
\left\{\begin{array}{l}
u_{t}-\operatorname{div} A(x, t, u, \nabla u)=f \quad \text { in } \Omega_{T}, \\
u=0 \quad \text { on } \partial \Omega \times(0, T), \\
u(\cdot, 0)=u_{0} \quad \text { in } \Omega .
\end{array}\right.
$$

Here $\Omega$ is a bounded open subset of $\mathbb{R}^{N}$, with $N \geqslant 2$. Correspondingly, $\Omega_{T}:=$ $\Omega \times(0, T)$ is the parabolic cylinder over $\Omega$ of height $T>0$. We adopt a usual notation $u_{t}$ for the time derivative and $\nabla u$ for gradient with respect to the space variable. We let $2 N /(N+2)<p<N$. For the data related to problem (1.1), we consider

$$
\begin{aligned}
& u_{0} \in L^{2}(\Omega) \\
& f \in L^{p^{\prime}}\left(0, T, W^{-1, p^{\prime}}(\Omega)\right)
\end{aligned}
$$

We assume that

$$
A=A(x, t, u, \xi): \Omega_{T} \times \mathbb{R} \times \mathbb{R}^{N} \rightarrow \mathbb{R}^{N}
$$

is a Carathéodory function (i.e. $A$ is measurable w.r.t. $(x, t) \in \Omega_{T}$ for all $(u, \xi) \in \mathbb{R} \times \mathbb{R}^{N}$ and continuous w.r.t. $(u, \xi) \in \mathbb{R} \times \mathbb{R}^{N}$ for a.e. $\left.(x, t) \in \Omega_{T}\right)$ satisfying the following monotonicity and boundedness conditions

$$
\begin{aligned}
& A(x, t, u, \xi) \cdot \xi \geqslant \alpha|\xi|^{p}-(b(x, t)|u|)^{p}-H(x, t) \\
& {[A(x, t, u, \xi)-A(x, t, u, \eta)] \cdot(\xi-\eta)>0 \quad \text { if } \xi \neq \eta} \\
& |A(x, t, u, \xi)| \leqslant \beta|\xi|^{p-1}+(\tilde{b}(x, t)|u|)^{p-1}+K(x, t)
\end{aligned}
$$

for a.e. $(x, t) \in \Omega_{T}$ and for any $u \in \mathbb{R}$ and $\xi, \eta \in \mathbb{R}^{N}$. Here $\alpha, \beta$ are positive constants, while $H, K, b$ and $\tilde{b}$ are nonnegative measurable functions defined on $\Omega_{T}$ such that $H \in L^{1}\left(\Omega_{T}\right), K \in L^{p^{\prime}}\left(\Omega_{T}\right)$ and

$$
b, \tilde{b} \in L^{\infty}\left(0, T, L^{N, \infty}(\Omega)\right)
$$

Here $L^{N, \infty}(\Omega)$ denotes the Marcinkiewicz space (see Sect. 2.2 for the definition).

Taking into account all the assumptions above, we consider the following notion of solution.

Definition 1. A solution to problem (1.1) is a function

$$
u \in C^{0}\left([0, T], L^{2}(\Omega)\right) \cap L^{p}\left(0, T, W_{0}^{1, p}(\Omega)\right)
$$

such that

$$
\begin{aligned}
& -\int_{0}^{T} \int_{\Omega} u \varphi_{t} \mathrm{~d} x \mathrm{~d} s+\int_{0}^{T} \int_{\Omega} A(x, s, u, \nabla u) \cdot \nabla \varphi \mathrm{d} x \mathrm{~d} s \\
& =\int_{\Omega} u_{0} \varphi(x, 0) \mathrm{d} x+\int_{0}^{T}\langle f, \varphi\rangle \mathrm{d} s
\end{aligned}
$$


for every $\varphi \in C^{\infty}\left(\bar{\Omega}_{T}\right)$ such that $\operatorname{supp} \varphi \subset[0, T) \times \Omega$.

Moreover, if $u \in C_{\mathrm{loc}}^{0}\left([0, \infty), L^{2}(\Omega)\right) \cap L_{\mathrm{loc}}^{p}\left(0, \infty, W_{0}^{1, p}(\Omega)\right)$ and the above holds true for all $T>0$, then $u$ is called a solution to problem (1.1) in $\Omega \times(0, \infty)$.

When the growth coefficient $b$ and $\tilde{b}$ are identically zero, the existence of a solution in the sense of Definition 1 can be found in $[15,16,23]$.

For the notation related to parabolic type function spaces such as $L^{p}\left(0, T, W_{0}^{1, p}(\Omega)\right)$ or similar, we refer the reader to Sect. 2 below. Above and throughout the paper, we denote by $\xi \cdot \eta$ the scalar product of two vectors $\xi, \eta \in \mathbb{R}^{N}$ and we denote by $\langle\cdot, \cdot\rangle$ the duality between $W^{-1, p^{\prime}}(\Omega)$ and $W_{0}^{1, p}(\Omega)$.

Our model equation is

$$
u_{t}-\operatorname{div}\left[\mu|\nabla u|^{p-2} \nabla u+|u|^{p-2} u\left(h(t) \frac{x}{|x|^{p}}+B(x, t)\right)\right]=f
$$

where $\mu>0, h \in L^{\infty}(0, T), B \in L^{\infty}\left(\Omega_{T}, \mathbb{R}^{N}\right)$ and $\Omega$ is the unit ball centered at the origin.

Our structure assumptions allow for parabolic equations with unbounded coefficients in the lower order term. We remark that the boundedness of the growth coefficients $b$ and $\tilde{b}$ is too restrictive in many applications as, for instance, in the case of the diffusion model for semiconductor devices (see [6]). We also recall that, in the linear homogeneous case, problem (1.1) describes the evolution of some Brownian motion and it is also known as Fokker-Planck equation (see $[5,21]$ and the references therein). On the other hand, a low integrability assumption for the term $b$ and $\tilde{b}$ does not guarantee the existence of a distributional solution in the sense of definition (1.8). In this case, other definitions of solutions have been introduced (see [3,21]). Assumption (1.7), in view of Sobolev embedding theorem (see Theorem 2.1 below), guarantees that

$$
\int_{\Omega_{T}} A(x, t, u, \nabla u) \cdot \nabla u \mathrm{~d} x \mathrm{~d} t<\infty
$$

that is, a solution in the sense of Definition 1 has finite energy.

Our existence result reads as follows.

Theorem 1.1. Let assumptions (1.2)-(1.7) be in charge. Assume further that

$$
\mathscr{D}_{b}:=\operatorname{dist}_{L^{\infty}\left(0, T ; L^{N, \infty}(\Omega)\right)}\left(b, L^{\infty}\left(\Omega_{T}\right)\right)<\frac{\alpha^{1 / p}}{S_{N, p}}
$$

where $S_{N, p}=\omega_{N}^{-1 / N} \frac{p}{N-p}$ is the Sobolev constant. Then problem (1.1) admits a solution.

Here $\omega_{N}$ denotes the measure of the unit ball in $\mathbb{R}^{N}$. In (1.10) $\operatorname{dist}_{L^{\infty}\left(0, T ; L^{N, \infty}(\Omega)\right)}\left(b, L^{\infty}\left(\Omega_{T}\right)\right)$ denotes the distance from bounded functions of the function $b$ with respect to the norm in $L^{\infty}\left(0, T ; L^{N, \infty}(\Omega)\right.$ ) (see formula (2.9) below for the definition). 
Condition (1.10), for the first time introduced in [12] and in [13], does not imply a smallness condition on the norm. In particular, (1.10) holds true whenever $1 \leqslant q<\infty$ and the coefficients $b$ and $\tilde{b}$ belong to $L^{\infty}\left(0, T, L^{N, q}(\Omega)\right)$, since $L^{\infty}(\Omega)$ is dense in $L^{N, q}(\Omega)$. Here $L^{N, q}(\Omega)$ denotes the Lorentz space (see Sect. 2.2 for the definition). We do not know if condition (1.10) is optimal in our framework. Nevertheless, in the elliptic counterpart of Theorem 1.1 (considered in [9]) such a condition turns to be optimal at least for $p=2$.

In example (1.9), condition (1.10) just gives a bound on $\|h\|_{L^{\infty}(0, T)}$ (see Remark 5.1 below), i.e.

$$
\|h\|_{L^{\infty}(0, T)}<\mu\left(\frac{N-p}{p}\right)^{p-1}
$$

We also study the behavior on time of a weak solution given in Theorem 1.1. More precisely, we estimate on time the $L^{2}$-norm of $u$ with the solution of a Cauchy problem related to a o.d.e. (see Theorem 6.1). As a consequence we provide estimates that highlight the different decay behavior as the exponent $p$ varies when $T=\infty$. The presence of the lower order term does not affect the decay to zero of the $L^{2}$-norm as $T$ goes to infinity (Corollary 6.2 below). Time decay for solutions to parabolic problems in absence of the lower order term can be found in $[10,14,18,22]$ and the references therein.

The novelty of the paper lies on the fact that in Theorem 1.1 above and Theorem 6.1 below we consider a family of operators not coercive with a singular growth coefficient in the lower order term. We recall that bounded functions are not dense in the Marcinkiewicz space $L^{N, \infty}(\Omega)$. In order to find a solution to (1.1), the main tool is an a priori estimate that could have interest by itself (see Proposition 3.2). Thanks to Leray-Schauder fixed point theorem, we first solve the problem in the case of bounded coefficients. Then, we obtain a solution to (1.1) as a limit of a sequence of solutions to suitable approximating problems. A solution in Theorem 1.1 satisfies an energy equality and then by using a recent result of [10] we are able to describe its asymptotic behavior.

\section{Preliminary results}

\subsection{Basic notation}

We will denote by $C$ (or by similar symbols such as $C_{1}, C_{2}, \ldots$ ) a generic positive constant, which may possibly vary from line to line. The dependence of $C$ upon various parameters will be highlighted in parentheses, adopting a notation of the type $C(\cdot, \ldots, \cdot)$.

\subsection{Function spaces}

Let $\Omega$ be a bounded domain in $\mathbb{R}^{N}$. Given $1<p<\infty$ and $1 \leqslant q<\infty$, the Lorentz space $L^{p, q}(\Omega)$ consists of all measurable functions $f$ defined on $\Omega$ for which the quantity

$$
\|f\|_{p, q}^{q}:=p \int_{0}^{\infty}\left[\lambda_{f}(k)\right]^{\frac{q}{p}} k^{q-1} \mathrm{~d} k
$$


is finite, where $\lambda_{f}(k):=|\{x \in \Omega:|f(x)|>k\}|$ is the distribution function of $f$. Note that $\|\cdot\|_{p, q}$ is equivalent to a norm and $L^{p, q}(\Omega)$ becomes a Banach space when endowed with it (see $[2,20])$. For $p=q$, the Lorentz space $L^{p, p}(\Omega)$ reduces to the Lebesgue space $L^{p}(\Omega)$. For $q=\infty$, the class $L^{p, \infty}(\Omega)$ consists of all measurable functions $f$ defined on $\Omega$ such that

$$
\|f\|_{p, \infty}^{p}:=\sup _{k>0} k^{p} \lambda_{f}(k)<\infty
$$

and it coincides with the Marcinkiewicz class, weak- $L^{p}(\Omega)$.

For Lorentz spaces the following inclusions hold

$$
L^{r}(\Omega) \subset L^{p, q}(\Omega) \subset L^{p, r}(\Omega) \subset L^{p, \infty}(\Omega) \subset L^{q}(\Omega),
$$

whenever $1 \leqslant q<p<r \leqslant \infty$. Moreover, for $1<p<\infty, 1 \leqslant q \leqslant \infty$ and $\frac{1}{p}+\frac{1}{p^{\prime}}=1, \frac{1}{q}+\frac{1}{q^{\prime}}=1$, if $f \in L^{p, q}(\Omega), g \in L^{p^{\prime}, q^{\prime}}(\Omega)$ we have the Hölder-type inequality

$$
\int_{\Omega}|f(x) g(x)| \mathrm{d} x \leqslant\|f\|_{p, q}\|g\|_{p^{\prime}, q^{\prime}} .
$$

As it is well known, $L^{\infty}(\Omega)$ is not dense in $L^{p, \infty}(\Omega)$. For a function $f \in L^{p, \infty}(\Omega)$ we define

$$
\operatorname{dist}_{L^{p, \infty}(\Omega)}\left(f, L^{\infty}(\Omega)\right)=\inf _{g \in L^{\infty}(\Omega)}\|f-g\|_{L^{p, \infty}(\Omega)} .
$$

In order to characterize the quantity in (2.3) we introduce for all $m>0$ the truncation operator at levels $\pm m$, namely

$$
\mathcal{T}_{m} y:=\min \{m, \max \{-m, y\}\} \quad \text { for } y \in \mathbb{R} .
$$

It is easy to verify that

$$
\lim _{m \rightarrow+\infty}\left\|f-\mathcal{T}_{m} f\right\|_{p, \infty}=\operatorname{dist}_{L^{p, \infty}(\Omega)}\left(f, L^{\infty}(\Omega)\right) .
$$

Clearly, for $1 \leqslant q<\infty$ any function in $L^{p, q}(\Omega)$ has vanishing distance to $L^{\infty}(\Omega)$. Indeed, $L^{\infty}(\Omega)$ is dense in $L^{p, q}(\Omega)$, the latter being continuously embedded into $L^{p, \infty}(\Omega)$.

Assuming that $0 \in \Omega$, a typical element of $L^{N, \infty}(\Omega)$ is $b(x)=b_{0} /|x|$, with $b_{0}$ a positive constant. An elementary calculation shows that

$$
\operatorname{dist}_{L^{N, \infty}(\Omega)}\left(b, L^{\infty}(\Omega)\right)=b_{0} \omega_{N}^{1 / N}
$$

where $\omega_{N}$ stands for the Lebesgue measure of the unit ball of $\mathbb{R}^{N}$.

We can also show that

$$
\operatorname{dist}_{L^{p, \infty}(\Omega)}\left(f, L^{\infty}(\Omega)\right)=\lim _{m \rightarrow+\infty}\left\|f \chi_{\{|f|>m\}}\right\|_{p, \infty} .
$$

To this end, it suffices to note the inequality

$$
|f-g| \geqslant\left(1-\frac{\|g\|_{\infty}}{m}\right)|f| \chi_{\{|f|>m\}}
$$

which holds for any $g \in L^{\infty}(\Omega)$ and $m>\|g\|_{\infty}$. For example, (2.6) implies that, if $\sigma p>1$, then for a positive function $f$

$$
\operatorname{dist}_{L^{p, \infty}(\Omega)}\left(f^{\sigma}, L^{\infty}(\Omega)\right)=\left[\operatorname{dist}_{L^{\sigma p, \infty}(\Omega)}\left(f, L^{\infty}(\Omega)\right)\right]^{\sigma} .
$$


The Sobolev embedding theorem in Lorentz spaces $[1,20]$ reads as

Theorem 2.1. Let us assume that $1<p<N, 1 \leqslant q \leqslant p$, then every function $u \in W_{0}^{1,1}(\Omega)$ verifying $|\nabla u| \in L^{p, q}(\Omega)$ actually belongs to $L^{p^{*}, q}(\Omega)$, where $p^{*}:=\frac{N p}{N-p}$ is the Sobolev exponent of $p$ and

$$
\|u\|_{p^{*}, q} \leqslant S_{N, p}\|\nabla u\|_{p, q}
$$

where $S_{N, p}$ is the Sobolev constant given by $S_{N, p}=\omega_{N}^{-1 / N} \frac{p}{N-p}$.

For our purposes, we also need to introduce some spaces involving the time variable. Hereafter, for the time derivative $u_{t}$ of a function $u$ we adopt the alternative notation $\partial_{t} u, \dot{u}, u^{\prime}$ or $\mathrm{d} u / \mathrm{d} t$. Let $T>0$. If we let $X$ be a Banach space endowed with a norm $\|\cdot\|_{X}$, the space $L^{p}(0, T, X)$ is defined as the class of all measurable functions $u:[0, T] \rightarrow X$ such that

$$
\|u\|_{L^{p}(0, T, X)}:=\left(\int_{0}^{T}\|u(t)\|_{X}^{p} \mathrm{~d} t\right)^{1 / p}<\infty
$$

whenever $1 \leqslant p<\infty$, and

$$
\|u\|_{L^{\infty}(0, T, X)}:=\underset{0<t<T}{\operatorname{ess} \sup _{0}}\|u(t)\|_{X}<\infty
$$

for $p=\infty$. Similarly, the space $C^{0}([0, T], X)$ represents the class of all continuous functions $u:[0, T] \rightarrow X$ with the norm

$$
\|u\|_{C^{0}([0, T], X)}:=\max _{0 \leqslant t \leqslant T}\|u(t)\|_{X}
$$

We will mainly deal with the case where $X$ is either a Lorentz space or Sobolev space $W_{0}^{1, p}(\Omega)$ equipped with the norm $\|g\|_{W_{0}^{1, p}(\Omega)}:=\|\nabla g\|_{L^{p}(\Omega)}$ for $g \in W_{0}^{1, p}(\Omega)$.

For $f \in L^{\infty}\left(0, T ; L^{p, \infty}(\Omega)\right)$ we define

$$
\operatorname{dist}_{L^{\infty}\left(0, T ; L^{p, \infty}(\Omega)\right)}\left(f, L^{\infty}\left(\Omega_{T}\right)\right)=\inf _{g \in L^{\infty}\left(\Omega_{T}\right)}\|f-g\|_{L^{\infty}\left(0, T ; L^{p, \infty}(\Omega)\right)}
$$

and as in (2.6) we find

$$
\operatorname{dist}_{L^{\infty}\left(0, T ; L^{p, \infty}(\Omega)\right)}\left(f, L^{\infty}\left(\Omega_{T}\right)\right)=\lim _{m \rightarrow+\infty}\left\|f \chi_{\{|f|>m\}}\right\|_{L^{\infty}\left(0, T ; L^{p, \infty}(\Omega)\right)} .
$$

We recall a well known result (see [23, Proposition 1.2, Chapter III, pag. 106]) involving the class of functions $W_{p}(0, T)$ defined as follows

$$
W_{p}(0, T):=\left\{v \in L^{p}\left(0, T, W_{0}^{1, p}(\Omega)\right): v_{t} \in L^{p^{\prime}}\left(0, T, W^{-1, p^{\prime}}(\Omega)\right)\right\}
$$

equipped with the norm

$$
\|u\|_{W_{p}(0, T)}:=\|u\|_{L^{p}\left(0, T, W_{0}^{1, p}(\Omega)\right)}+\left\|u_{t}\right\|_{L^{p^{\prime}}\left(0, T, W^{-1, p^{\prime}}(\Omega)\right)}
$$


Lemma 2.2. Let $p>2 N /(N+2)$. Then $W_{p}(0, T)$ is contained into $C^{0}\left([0, T], L^{2}(\Omega)\right)$ and any function $u \in W_{p}(0, T)$ satisfies

$$
\|u\|_{C^{0}\left([0, T], L^{2}(\Omega)\right)} \leqslant C\|u\|_{W_{p}(0, T)}
$$

for some constant $C>0$.

Furthermore, the function $t \in[0, T] \mapsto\|u(\cdot, t)\|_{L^{2}(\Omega)}^{2}$ is absolutely continuous and

$$
\frac{1}{2} \frac{\mathrm{d}}{\mathrm{d} t}\|u(\cdot, t)\|_{L^{2}(\Omega)}^{2}=\left\langle u_{t}(\cdot, t), u(\cdot, t)\right\rangle \quad \text { for a.e. } t \in[0, T]
$$

Finally, we recall the classical compactness result due to Aubin-Lions (see [23, Proposition 1.3, Chapter III, pag. 106]).

Lemma 2.3. (Aubin-Lions) Let $X_{0}, X, X_{1}$ be Banach spaces with $X_{0}$ and $X_{1}$ reflexive. Assume that $X_{0}$ is compactly embedded into $X$ and $X$ is continuosly embedded into $X_{1}$. For $1<p, q<\infty$ let

$$
W:=\left\{u \in L^{p}\left(0, T, X_{0}\right): \partial_{t} u \in L^{q}\left(0, T, X_{1}\right)\right\}
$$

Then $W$ is compactly embedded into $L^{p}(0, T, X)$.

A prototypical example of application of this lemma corresponds to the choices $q=p, X_{0}=W_{0}^{1, p}(\Omega), X_{1}=W^{-1, p^{\prime}}(\Omega)$ and $X=L^{p}(\Omega)$ if $p \geqslant 2$ or $X=L^{2}(\Omega)$ for $\frac{2 N}{N+2}<p<2$. Obviously $L^{2}(\Omega) \subset L^{p}(\Omega)$ as long as $p<2$, and therefore we deduce the following.

Lemma 2.4. If $p>2 N /(N+2)$ then $W_{p}(0, T)$ is compactly embedded into $L^{p}\left(\Omega_{T}\right)$.

\subsection{Gronwall type results}

We recall a couple of lemmas (see [10]) whose application will be essential in the study of the time behavior of the solutions to (1.1).

Lemma 2.5. Consider a Carathéodory function $\psi:\left[t_{0}, T\right] \times \mathbb{R} \rightarrow \mathbb{R}_{+}$such that for every $r>0$ there exists $k_{r} \in L^{1}\left(t_{0}, T ; \mathbb{R}_{+}\right)$satisfying for a.e. $t \in\left[t_{0}, T\right]$

$$
\sup _{|x| \leqslant r} \psi(t, x) \leqslant k_{r}(t) \text {. }
$$

Let $g \in L^{1}\left(t_{0}, T ; \mathbb{R}\right)$ and $\gamma:\left[t_{0}, T\right] \rightarrow \mathbb{R}_{+}$be measurable, bounded and satisfy

$$
\gamma\left(t_{2}\right)-\gamma\left(t_{1}\right)+\int_{t_{1}}^{t_{2}} \psi(t, \gamma(t)) \mathrm{d} t \leqslant \int_{t_{1}}^{t_{2}} g(t) \mathrm{d} t \quad t_{0} \leqslant t_{1} \leqslant t_{2} \leqslant T .
$$

Then there exists a solution $x(\cdot) \in W^{1,1}\left(\left[t_{0}, T\right]\right)$ of the Cauchy problem

$$
\left\{\begin{array}{l}
x^{\prime}(t)=-\psi(t, x(t))+g(t), \quad \text { a.e. } t \in\left[t_{0}, T\right] \\
x\left(t_{0}\right)=\gamma\left(t_{0}\right)
\end{array}\right.
$$

such that $\gamma(t) \leqslant x(t)$ for all $t \in\left[t_{0}, T\right]$. 
Furthermore, if $g \in L^{1}\left(t_{0}, \infty ; \mathbb{R}\right), \psi$ is defined on $\left[t_{0},+\infty\right) \times \mathbb{R}, \gamma:\left[t_{0},+\infty\right) \rightarrow$ $\mathbb{R}_{+}$is measurable and locally bounded and for every $T>t_{0}$ the above assumptions hold true, then there exists a solution $x$ to $(2.13)$ defined on $\left[t_{0}, \infty\right)$ such that $\gamma \leqslant x$. In particular,

$$
\limsup _{t \rightarrow \infty} \gamma(t) \leqslant \limsup _{t \rightarrow \infty} x(t) .
$$

Lemma 2.6. Under all the assumptions of Lemma 2.5 suppose that $\psi(t, a)=0$ for all $a \leqslant 0, g(\cdot) \geq 0, \psi(t, \cdot)$ is increasing for a.e. $t \in\left[t_{0}, T\right]$ and that for any $R>r>0$ there exists $\bar{k}_{R, r} \in L^{1}\left(t_{0}, T ; \mathbb{R}_{+}\right)$satisfying for a.e. $t \in\left[t_{0}, T\right]$

$$
|\psi(t, x)-\psi(t, y)| \leqslant k_{R, r}(t)|x-y| \quad \forall x, y \in[r, R] .
$$

Then the solution $z(\cdot)$ of

$$
\left\{\begin{array}{l}
z^{\prime}(t)=-\psi(t, z(t)), \quad \text { a.e. } t \in\left[t_{0}, T\right] \\
z\left(t_{0}\right)=\gamma\left(t_{0}\right)
\end{array}\right.
$$

is unique and well defined on $\left[t_{0}, T\right], z(\cdot) \geq 0$ and $\gamma(t) \leqslant x(t) \leqslant z(t)+$ $\int_{t_{0}}^{t} g(s) \mathrm{d} s$ for all $t \in\left[t_{0}, T\right]$, where $x(\cdot)$ is as in the claim of Lemma 2.5.

\section{Weak type and a priori estimates for an auxiliary problem}

An a priori estimate on the distribution function of a solution to problem (1.1) will be fundamental in order to prove Theorem 1.1. To this aim, we let $\phi: \mathbb{R} \rightarrow \mathbb{R}$ be the function defined as

$$
\phi(w):=\frac{1}{p-1}\left[1-\frac{1}{(1+|w|)^{p-1}}\right] \operatorname{sign}(w) \text { for } w \in \mathbb{R} \backslash\{0\} \text { and } \phi(0):=0
$$

We set $\Phi(w):=\int_{0}^{|w|} \phi(\rho) d \rho$ and $\Psi:(0, \infty) \rightarrow(0, \infty)$ be the reciprocal of the restriction of $\Phi$ to $(0, \infty)$, so that it is a continuous and decreasing function such that $\Psi(k) \rightarrow 0$ as $k \rightarrow \infty$. With this notation at hand, we have the following result.

Lemma 3.1. Let assumptions (1.2)-(1.4) and (1.6) be in charge and $b, \tilde{b} \in$ $L^{\infty}\left(0, T, L^{N, \infty}(\Omega)\right)$. For a fixed $\lambda \in(0,1]$, assume that the problem

$$
\left\{\begin{array}{l}
\frac{u_{t}}{\lambda}-\operatorname{div} A\left(x, t, u, \frac{\nabla u}{\lambda}\right)=f \quad \text { in } \Omega_{T}, \\
u=0 \quad \text { on } \partial \Omega \times(0, T), \\
u(\cdot, 0)=\lambda u_{0} \quad \text { in } \Omega,
\end{array}\right.
$$

admits a solution $u \in C^{0}\left([0, T], L^{2}(\Omega)\right) \cap L^{p}\left(0, T, W_{0}^{1, p}(\Omega)\right)$. Then, for every $k>0$ we have

$$
\sup _{0<t<T}|\{x \in \Omega:|u(x, t)|>k\}| \leqslant \Psi(k) M_{0}
$$


where

$$
M_{0}=\frac{1}{2}\left\|u_{0}\right\|_{L^{2}(\Omega)}^{2}+\|H\|_{L^{1}\left(\Omega_{T}\right)}+\|b\|_{L^{p}\left(\Omega_{T}\right)}^{p}+\alpha^{-1 /(p-1)}\|f\|_{L^{p^{\prime}}\left(0, T, W^{-1, p^{\prime}}(\Omega)\right)}^{p^{\prime}}
$$

Proof. First of all, we set $w:=u / \lambda$, in such a way that $w$ solves the problem

$$
\left\{\begin{array}{l}
w_{t}-\operatorname{div} A(x, t, \lambda w, \nabla w)=f \quad \text { in } \Omega_{T}, \\
w=0 \quad \text { on } \partial \Omega \times(0, T), \\
w(\cdot, 0)=u_{0} \quad \text { in } \Omega,
\end{array}\right.
$$

We fix $t \in(0, T)$ and we choose $\varphi:=\phi(w) \chi_{(0, t)}$ as a test function for (3.4). (This can be done since $\phi$ is a Lipschitz function in the whole of $\mathbb{R}$.) In this way, we have

$\int_{\Omega} \Phi(w) \mathrm{d} x+\int_{\Omega_{t}} A(x, s, \lambda w, \nabla w) \cdot \nabla \phi(w) \mathrm{d} x \mathrm{~d} s=\int_{\Omega} \Phi\left(u_{0}\right) \mathrm{d} x+\int_{0}^{t}\langle f, \phi(w)\rangle \mathrm{d} s$,

where $\Omega_{t}=\Omega \times(0, t)$. Observe explicitly that

$$
\nabla \phi(w)=\frac{\nabla w}{(1+|w|)^{p}} \chi_{(0, t)}
$$

Therefore, by (1.4) we have

$$
\begin{aligned}
& \int_{\Omega} \Phi(w) \mathrm{d} x+\alpha \int_{\Omega_{t}} \frac{|\nabla w|^{p}}{(1+|w|)^{p}} \mathrm{~d} x \mathrm{~d} s \\
& \quad \leqslant \int_{\Omega} \Phi\left(u_{0}\right) \mathrm{d} x+\int_{0}^{t}\langle f, \phi(w)\rangle \mathrm{d} s+\int_{\Omega_{t}}\left(\frac{b|w|}{1+|w|}\right)^{p} \mathrm{~d} x \mathrm{~d} s+\int_{\Omega_{t}} H(x, s) \mathrm{d} x \mathrm{~d} s
\end{aligned}
$$

By means of Young inequality we have

$$
\int_{0}^{t}\langle f, \phi(w)\rangle \mathrm{d} s \leqslant \alpha \int_{\Omega_{t}} \frac{|\nabla w|^{p}}{(1+|w|)^{p}} \mathrm{~d} x \mathrm{~d} s+\alpha^{-1 /(p-1)}\|f\|_{L^{p^{\prime}}\left(0, T, W^{-1, p^{\prime}}(\Omega)\right)}^{p^{\prime}}
$$

In view of the latter estimate and of the fact that $0 \leqslant \Phi(k) \leqslant \frac{1}{2} k^{2}$ for any $k \geqslant 0$, from (3.6) we infer

$$
\int_{\Omega} \Phi(w) \mathrm{d} x \leqslant M_{0}
$$

For $t \in(0, T)$ fixed, we set $E_{k}(t):=\{x \in \Omega:|u(x, t)|>k\}$ for $k>0$. Since $\lambda \in[0,1]$, clearly $E_{k}(t) \subset\{x \in \Omega:|w(x, t)|>k\}$. By the monotonicity of $\Phi$ and by (3.8), we have $\left|E_{k}(t)\right| \Phi(k) \leqslant M_{0}$, which implies the claimed estimate.

Now, we are in position to prove some a priori estimate for a solution to problem (3.1). 
Proposition 3.2. Let assumptions of Lemma 3.1 be fulfilled and assume that condition (1.10) holds true. For a fixed $\lambda \in(0,1]$, any solution $u \in$ $C^{0}\left([0, T], L^{2}(\Omega)\right) \cap L^{p}\left(0, T, W_{0}^{1, p}(\Omega)\right)$ to problem (3.1) satisfies the following estimate

$$
\sup _{0<t<T} \int_{\Omega}|u(\cdot, t)|^{2} \mathrm{~d} x+\int_{\Omega_{T}}|\nabla u|^{p} \mathrm{~d} x \mathrm{~d} t \leqslant C
$$

for some positive constant $C$ depending only on $N, p, \alpha,\|b\|_{L^{p}\left(\Omega_{T}\right)}, \mathscr{D}_{b}$, $\left\|u_{0}\right\|_{L^{2}(\Omega)},\|H\|_{L^{1}\left(\Omega_{T}\right)},\|f\|_{L^{p^{\prime}}\left(0, T, W^{-1, p^{\prime}}(\Omega)\right)} \cdot$

Proof. We set $w:=u / \lambda$, in such a way that $w$ solves the problem (3.4). We fix $t \in(0, T)$ and we choose $\varphi:=w \chi_{(0, t)}$ as a test function for (3.4) so we get $\frac{1}{2}\|w(\cdot, t)\|_{L^{2}(\Omega)}^{2}+\int_{\Omega_{t}} A(x, s, \lambda w, \nabla w) \cdot \nabla w \mathrm{~d} x \mathrm{~d} s=\frac{1}{2}\left\|u_{0}\right\|_{L^{2}(\Omega)}^{2}+\int_{0}^{t}\langle f, w\rangle \mathrm{d} s$

By means of Young inequality we have for $0<\varepsilon<1$

$$
\int_{0}^{t}\langle f, w\rangle \mathrm{d} s \leqslant \varepsilon \int_{\Omega_{t}}|\nabla w|^{p} \mathrm{~d} x \mathrm{~d} s+C(\varepsilon, p)\|f\|_{L^{p^{\prime}}\left(0, T, W^{-1, p^{\prime}}(\Omega)\right)}^{p^{\prime}}
$$

By (1.4) we further have

$$
\begin{aligned}
\frac{1}{2}\|w(\cdot, t)\|_{L^{2}(\Omega)}^{2} & +\alpha \int_{\Omega_{t}}|\nabla w|^{p} \mathrm{~d} x \mathrm{~d} s \leqslant \frac{1}{2}\left\|u_{0}\right\|_{L^{2}(\Omega)}^{2}+\varepsilon \int_{\Omega_{t}}|\nabla w|^{p} \mathrm{~d} x \mathrm{~d} s \\
& +C(\varepsilon, p)\|f\|_{L^{p^{\prime}}\left(0, T, W^{-1, p^{\prime}(\Omega)}\right)}^{p^{\prime}}+\int_{\Omega_{t}}(\lambda b|w|)^{p} \mathrm{~d} x \mathrm{~d} s(3 . \\
& +\int_{\Omega_{t}} H(x, s) \mathrm{d} x \mathrm{~d} s
\end{aligned}
$$

Now, we provide an estimate on

$$
\int_{\Omega_{t}}(\lambda b|w|)^{p} \mathrm{~d} x \mathrm{~d} s=\int_{\Omega_{t}}(b|u|)^{p} \mathrm{~d} x \mathrm{~d} s
$$

For $m>0$ to be chosen later, we write

$$
\int_{\Omega_{t}}(b|u|)^{p} \mathrm{~d} x \mathrm{~d} s=\int_{\Omega_{t}}\left(b \chi_{\{b \leqslant m\}}|u|\right)^{p} \mathrm{~d} x \mathrm{~d} s+\int_{\Omega_{t}}\left(b \chi_{\{b>m\}}|u|\right)^{p} \mathrm{~d} x \mathrm{~d} s
$$

We estimate separately the two terms. For $k>0$ fixed, we have

$$
\int_{\Omega_{t}}\left(b \chi_{\{b \leqslant m\}}|u|\right)^{p} \mathrm{~d} x \mathrm{~d} s \leqslant m^{p} \int_{0}^{t} \mathrm{~d} s \int_{\{|u(\cdot, s)|>k\}}|u|^{p} \mathrm{~d} x+k^{p} \int_{0}^{t} \mathrm{~d} s \int_{\Omega} b(x, s)^{p} \mathrm{~d} x
$$


In particular, we apply Hölder inequality (2.2), Sobolev inequality (2.8) slicewise and Lemma 3.1 to get

$$
\begin{aligned}
\int_{0}^{t} \mathrm{~d} s \int_{\{|u(\cdot, s)|>k\}}|u|^{p} \mathrm{~d} x & =\int_{0}^{t} \mathrm{~d} s \int_{\Omega}\left|u \chi_{\{|u(\cdot, s)|>k\}}\right|^{p} \mathrm{~d} x \\
& \leqslant \int_{0}^{t}\left\|\chi_{\{|u(\cdot, s)|>k\}}\right\|_{L^{N, \infty}(\Omega)}^{p}\|u\|_{L^{p^{*}, p}(\Omega)}^{p} \mathrm{~d} s \\
& \leqslant S_{N, p}^{p}\left(M_{0} \Psi(k)\right)^{p / N} \int_{\Omega_{t}}|\nabla u|^{p} \mathrm{~d} x \mathrm{~d} s
\end{aligned}
$$

where $M_{0}$ is the constant in (3.3). A similar combination of Hölder inequality and Sobolev inequality yields

$$
\int_{\Omega_{t}}\left(b \chi_{\{b>m\}}|u|\right)^{p} \mathrm{~d} x \mathrm{~d} s \leqslant S_{N, p}^{p}\left\|b \chi_{\{b>m\}}\right\|_{L^{\infty}\left(0, T, L^{N, \infty}(\Omega)\right)}^{p} \int_{\Omega_{t}}|\nabla u|^{p} \mathrm{~d} x \mathrm{~d} s
$$

Inserting (3.14), (3.15) and (3.16) into (3.13) we obtain

$$
\begin{aligned}
\int_{\Omega_{t}}(b|u|)^{p} \mathrm{~d} x \mathrm{~d} s \leqslant & {\left[S_{N, p}^{p} m^{p}\left(M_{0} \Psi(k)\right)^{p / N}\right.} \\
& \left.+S_{N, p}^{p}\left\|b \chi_{\{b>m\}}\right\|_{L^{\infty}\left(0, T, L^{N, \infty}(\Omega)\right)}^{p}\right]\|\nabla u\|_{L^{p}\left(\Omega_{t}\right)}^{p} \\
& +k^{p} \int_{0}^{t} \mathrm{~d} s \int_{\Omega} b(x, s)^{p} \mathrm{~d} x
\end{aligned}
$$

Observe that (3.12) and (3.17) imply

$$
\begin{aligned}
& \frac{1}{2}\|w(\cdot, t)\|_{L^{2}(\Omega)}^{2}+\alpha\|\nabla w\|_{L^{p}\left(\Omega_{t}\right)}^{p} \leqslant \frac{1}{2}\left\|u_{0}\right\|_{L^{2}(\Omega)}^{2}+k^{p}\|b\|_{L^{p}\left(\Omega_{T}\right)}^{p} \\
& +C(\varepsilon, p)\|f\|_{L^{p^{\prime}}\left(0, T, W^{-1, p^{\prime}(\Omega)}\right)}^{p^{\prime}} \\
& \quad+\|H\|_{L^{1}\left(\Omega_{T}\right)}+\left[\varepsilon+S_{N, p}^{p} m^{p}\left(M_{0} \Psi(k)\right)^{p / N}\right. \\
& \left.\quad+S_{N, p}^{p}\left\|b \chi_{\{b>m\}}\right\|_{L^{\infty}\left(0, T, L^{N, \infty}(\Omega)\right)}^{p}\right]\|\nabla w\|_{L^{p}\left(\Omega_{t}\right)}^{p}
\end{aligned}
$$

We are able to reabsorb in the left hand side the last term by choosing properly $m, k$ and $\varepsilon$. First, it is sufficient to have $m$ so large to guarantee

$$
S_{N, p}^{p}\left\|b \chi_{\{b>m\}}\right\|_{L^{\infty}\left(0, T, L^{N, \infty}(\Omega)\right)}^{p}<\alpha
$$

and the existence of such a value of $m$ is a direct consequence of (1.10) and the characterization of distance in (2.10). A proper choice of $k$ and $\varepsilon$ can be performed coherently with (3.19) and taking into account the properties of $\Psi(\cdot)$. In particular, denoting by $C$ a constant depending only on $N, p, \alpha$ and on $\|b\|_{L^{p}\left(\Omega_{T}\right)}, \mathscr{D}_{b},\left\|u_{0}\right\|_{L^{2}(\Omega)},\|H\|_{L^{1}\left(\Omega_{T}\right)},\|f\|_{L^{p^{\prime}}\left(0, T, W^{-1, p^{\prime}}(\Omega)\right)}$, by combining (3.18) and (3.19) we deduce

$$
\|w(\cdot, t)\|_{L^{2}(\Omega)}^{2}+\|\nabla w\|_{L^{p}\left(\Omega_{t}\right)}^{p} \leqslant C
$$

Taking into account $(3.20)$ and recalling that $\lambda \in(0,1]$, the latter estimate leads to the conclusion of the proof. 
Remark 3.3. We point out that the a priori estimate (3.9) is uniform with respect to the parameter $\lambda$.

\section{Parabolic equations with bounded coefficients}

This section is devoted to the proof of the existence of a solution to problem (1.1) in the special case $b, \tilde{b} \in L^{\infty}\left(\Omega_{T}\right)$. We shall use on this account the following version of Leray-Schauder fixed point theorem as in (see [11, Theorem 11.3 pg. 280]).

Theorem 4.1. Let $\mathcal{F}$ be a compact mapping of a Banach space $X$ into itself, and suppose there exists a constant $M$ such that $\|x\|_{X}<M$ for all $x \in X$ and $\lambda \in[0,1]$ satisfying $x=\lambda \mathcal{F}(x)$. Then, $\mathcal{F}$ has a fixed point.

We recall that a continuous mapping between two Banach spaces is called compact if the images of bounded sets are precompact.

Accordingly, the main result of this section reads as follows.

Theorem 4.2. Let assumptions (1.2)-(1.6) be in charge and $b, \tilde{b} \in L^{\infty}\left(\Omega_{T}\right)$. Then problem (1.1) admits a solution.

Proof. We let $v \in L^{p}\left(\Omega_{T}\right)$, and consider the problem

$$
\left\{\begin{array}{l}
u_{t}-\operatorname{div} A(x, t, v, \nabla u)=f \quad \text { in } \Omega_{T}, \\
u=0 \quad \text { on } \partial \Omega \times(0, T), \\
u(\cdot, 0)=u_{0} \quad \text { in } \Omega,
\end{array}\right.
$$

Problem (4.1) admits a solution by the classical theory of pseudomonotone operators [16] and by the strict monotonicity of the vector field

$$
(x, t, \xi) \in \Omega_{T} \times \mathbb{R}^{N} \mapsto \bar{A}(x, t, \xi):=A(x, t, v, \xi)
$$

such a solution is unique. Hence, the map $\mathcal{F}$ which takes $v$ to the solution $u$ is well defined and certainly acts from $L^{p}\left(\Omega_{T}\right)$ into itself. Our goal is to determine a fixed point for $\mathcal{F}$, which is obviously a solution to (1.1). We want to apply Theorem 4.1, so we need to show that $\mathcal{F}: L^{p}\left(\Omega_{T}\right) \rightarrow L^{p}\left(\Omega_{T}\right)$ is continuous, compact and the set

$$
\mathcal{U}:=\left\{u \in L^{p}\left(\Omega_{T}\right): u=\lambda \mathcal{F}[u] \text { for some } \lambda \in[0,1]\right\}
$$

is bounded in $L^{p}\left(\Omega_{T}\right)$. First observe that the boundedness of $\mathcal{U}$ is a direct consequence of Proposition 3.2.

Let us prove that $\left\{\mathcal{F}\left[v_{n}\right]\right\}_{n \in \mathbb{N}}$ is a precompact sequence if $\left\{v_{n}\right\}_{n \in \mathbb{N}}$ is a bounded sequence in $L^{p}\left(\Omega_{T}\right)$. We need to show that $u_{n}:=\mathcal{F}\left[v_{n}\right]$ admits a subsequence strongly converging in $L^{p}\left(\Omega_{T}\right)$. By definition of $\mathcal{F}$, we see that 
$u_{n}$ solves the problem

$$
\left\{\begin{array}{l}
\partial_{t} u_{n}-\operatorname{div} A\left(x, t, v_{n}, \nabla u_{n}\right)=f \quad \text { in } \Omega_{T}, \\
u_{n}=0 \quad \text { on } \partial \Omega \times(0, T), \\
u_{n}(\cdot, 0)=u_{0} \quad \text { in } \Omega,
\end{array}\right.
$$

By testing the equation in (4.2) by $u_{n}$ itself and arguing similarly as before, we see that

$$
\begin{aligned}
\sup _{0<t<T} & \int_{\Omega}\left|u_{n}(\cdot, t)\right|^{2} \mathrm{~d} x+\int_{\Omega_{T}}\left|\nabla u_{n}\right|^{p} \mathrm{~d} x \mathrm{~d} t \\
& \leqslant C\left[\left\|u_{0}\right\|_{L^{2}(\Omega)}^{2}+\|H\|_{L^{1}\left(\Omega_{T}\right)}+\|b\|_{L^{\infty}\left(\Omega_{T}\right)}^{p}\left\|v_{n}\right\|_{L^{p}\left(\Omega_{T}\right)}^{p}+\|f\|_{L^{p^{\prime}}\left(0, T, W^{-1, p^{\prime}}(\Omega)\right)}^{p^{\prime}}\right]
\end{aligned}
$$

So in particular $\left\{\left|\nabla u_{n}\right|\right\}_{n \in \mathbb{N}}$ is bounded in $L^{p}\left(\Omega_{T}\right)$. Using the equation in (4.2) we see that $\left\{\partial_{t} u_{n}\right\}_{n \in \mathbb{N}}$ is bounded in $L^{p^{\prime}}\left(0, T, W^{-1, p^{\prime}}(\Omega)\right)$ and so $\left\{u_{n}\right\}_{n \in \mathbb{N}}$ has a subsequence that strongly converges in $L^{p}\left(\Omega_{T}\right)$ as a direct consequence of the Aubin-Lions lemma.

Let us prove the continuity of $\mathcal{F}$. Let $\left\{v_{n}\right\}_{n \in \mathbb{N}}$ be a strongly converging sequence in $L^{p}\left(\Omega_{T}\right)$, say

$$
v_{n} \rightarrow v \quad \text { in } L^{p}\left(\Omega_{T}\right) \text { strongly }
$$

We set $u_{n}:=\mathcal{F}\left[v_{n}\right]$. We already know that $\left\{u_{n}\right\}_{n \in \mathbb{N}}$ is compact sequence in $L^{p}\left(\Omega_{T}\right)$ and also that estimate (4.3) holds true. So there exists $u \in W_{p}(0, T)$ such that (for a subsequence not relabeled):

$$
\begin{array}{cc}
u_{n} \rightarrow u & \text { strongly in } L^{p}\left(\Omega_{T}\right) \\
\nabla u_{n} \rightarrow \nabla u & \text { weakly in } L^{p}\left(\Omega_{T}, \mathbb{R}^{N}\right) \\
u_{n} \rightarrow^{*} u & \text { weakly* in } L^{\infty}\left(0, T ; L^{2}(\Omega)\right) \\
\partial_{t} u_{n} \rightarrow \partial_{t} u & \text { weakly in } L^{p^{\prime}}\left(0, T, W^{-1, p^{\prime}}(\Omega)\right) .
\end{array}
$$

Observe further that $u \in C^{0}\left([0, T], L^{2}(\Omega)\right)$ with $u(\cdot, 0)=u_{0}$. It is a direct consequence of the inclusion of Lemma 2.2, the boundednes of $\left\{u_{n}\right\}_{n \in \mathbb{N}}$ in $W_{p}(0, T)$ and of the convergence $u_{n} \rightarrow u$ weakly in $L^{2}(\Omega)$ for all $t \in[0, T]$. In order to prove the continuity of $\mathcal{F}$, we need to show that

$$
u=\mathcal{F}[v]
$$

Again, we know that $u_{n}$ solves (4.2), namely for every $\varphi \in C^{\infty}\left(\bar{\Omega}_{T}\right)$ with support contained in $[0, T) \times \Omega$ we have

$$
\int_{0}^{T}\left\langle\partial_{t} u_{n}, \varphi\right\rangle \mathrm{d} t+\int_{0}^{T} \int_{\Omega} A\left(x, t, v_{n}, \nabla u_{n}\right) \cdot \nabla \varphi \mathrm{d} x \mathrm{~d} t=\int_{0}^{T}\langle f, \varphi\rangle \mathrm{d} t
$$


If we choose $u_{n}-u$ as a test function in the above identity we have

$$
\begin{aligned}
& \frac{1}{2}\left\|u_{n}(T)-u(T)\right\|_{L^{2}(\Omega)}^{2}+\int_{0}^{T}\left\langle\partial_{t} u, u_{n}-u\right\rangle \mathrm{d} t \\
& \quad+\int_{0}^{T} \int_{\Omega} A\left(x, t, v_{n}, \nabla u_{n}\right) \cdot \nabla\left(u_{n}-u\right) \mathrm{d} x \mathrm{~d} t=\int_{0}^{T}\left\langle f, u_{n}-u\right\rangle \mathrm{d} t
\end{aligned}
$$

Since $u_{n}-u \rightarrow 0$ weakly in $L^{p}\left(0, T ; W_{0}^{1, p}(\Omega)\right)$, it is clear that

$$
\int_{0}^{T}\left\langle\partial_{t} u, u_{n}-u\right\rangle \mathrm{d} t \rightarrow 0 \quad \text { as } n \rightarrow \infty
$$

and

$$
\int_{0}^{T}\left\langle f, u_{n}-u\right\rangle \mathrm{d} t \rightarrow 0 \quad \text { as } n \rightarrow \infty
$$

Being the first term in (4.10) nonnegative, we get

$$
\limsup _{n \rightarrow \infty} \int_{0}^{T} \int_{\Omega} A\left(x, t, v_{n}, \nabla u_{n}\right) \cdot \nabla\left(u_{n}-u\right) \mathrm{d} x \mathrm{~d} t \leqslant 0
$$

From the boundedness of $\tilde{b}$, the sequence $\left\{A\left(x, t, v_{n}, \nabla u\right)\right\}_{n \in \mathbb{N}}$ strongly converges in $L^{p^{\prime}}\left(\Omega_{T}\right)$ and so

$$
\lim _{n \rightarrow \infty} \int_{0}^{T} \int_{\Omega} A\left(x, t, v_{n}, \nabla u\right) \cdot \nabla\left(u_{n}-u\right) \mathrm{d} x \mathrm{~d} t=0
$$

The latter estimate, the strict monotonicity of $A$ and (4.11) give us

$$
\lim _{n \rightarrow \infty} \int_{0}^{T} \int_{\Omega} A\left(x, t, v_{n}, \nabla u_{n}\right) \cdot \nabla\left(u_{n}-u\right) \mathrm{d} x \mathrm{~d} t=0
$$

It is clear that $\left\{A\left(x, t, v_{n}, \nabla u_{n}\right)\right\}_{n \in \mathbb{N}}$ is bounded in $L^{p^{\prime}}\left(\Omega_{T}\right)$ and so it weakly converges in $L^{p^{\prime}}\left(\Omega_{T}\right)$ to some $\tilde{A}$. We use the Minty trick to recover that $\tilde{A}(x, t)=A(x, t, v(x, t), \nabla u(x, t))$ a.e. in $\Omega_{T}$. Namely, let $\eta \in L^{p}\left(\Omega_{T}, \mathbb{R}^{N}\right)$. Observe that

$$
\begin{aligned}
0 \leqslant & \int_{\Omega_{T}}\left[A\left(x, t, v_{n}, \nabla u_{n}\right)-A\left(x, t, v_{n}, \eta\right)\right] \cdot\left(\nabla u_{n}-\eta\right) \mathrm{d} x \mathrm{~d} t \\
= & \int_{\Omega_{T}}\left[A\left(x, t, v_{n}, \nabla u_{n}\right)-A\left(x, t, v_{n}, \nabla u\right)\right] \cdot\left(\nabla u_{n}-\nabla u\right) \mathrm{d} x \mathrm{~d} t \\
& +\int_{\Omega_{T}}\left[A\left(x, t, v_{n}, \nabla u\right)-A\left(x, t, v_{n}, \eta\right)\right] \cdot\left(\nabla u_{n}-\nabla u\right) \mathrm{d} x \mathrm{~d} t \\
& +\int_{\Omega_{T}}\left[A\left(x, t, v_{n}, \nabla u_{n}\right)-A\left(x, t, v_{n}, \eta\right)\right] \cdot(\nabla u-\eta) \mathrm{d} x \mathrm{~d} t
\end{aligned}
$$

Passing to the limit, we get

$$
0 \leqslant \int_{\Omega_{T}}[\tilde{A}(x, t)-A(x, t, v, \eta)] \cdot(\nabla u-\eta) \mathrm{d} x \mathrm{~d} t
$$


We choose $\eta:=\nabla u-\lambda \psi$ in (4.14) where $\psi \in L^{p}\left(\Omega_{T}, \mathbb{R}^{N}\right)$ and $\lambda \in \mathbb{R}$. Then

$$
\lambda \int_{\Omega_{T}}[\tilde{A}(x, t)-A(x, t, v, \nabla u-\lambda \psi)] \cdot \psi \mathrm{d} x \mathrm{~d} t \geqslant 0
$$

If we assume that $\lambda>0$, we divide by $\lambda$ itself and then letting $\lambda \rightarrow 0^{+}$we have

$$
\int_{\Omega_{T}}[\tilde{A}(x, t)-A(x, t, v, \nabla u)] \cdot \psi \mathrm{d} x \mathrm{~d} t \geqslant 0
$$

Arguing similarly if $\lambda<0$ we get the opposite inequality than (4.16), so we conclude that for every $\psi \in L^{p}\left(\Omega_{T}, \mathbb{R}^{N}\right)$

$$
\int_{\Omega_{T}}[\tilde{A}(x, t)-A(x, t, v, \nabla u)] \cdot \psi \mathrm{d} x \mathrm{~d} t=0
$$

i.e. $\tilde{A}(x, t)=A(x, t, v(x, t), \nabla u(x, t))$ a.e. in $\Omega_{T}$.

We are in position to pass to the limit in (4.9). Therefore

$$
-\int_{0}^{T} \int_{\Omega} u \partial_{t} \varphi \mathrm{d} s+\int_{0}^{T} \int_{\Omega} A(x, s, v, \nabla u) \cdot \nabla \varphi d x d s=\int_{\Omega} u_{0} \varphi(x, 0) \mathrm{d} x+\int_{0}^{T}\langle f, \varphi\rangle \mathrm{d} s
$$

Since for any subsequence of $u_{n}$ we can repeat previous argument and get (4.8), the limit $u$ being independent of the choice of the subsequence, we can conclude that $\mathcal{F}$ is continuous.

\section{Proof of the existence result via approximation scheme}

Proof of Theorem 1.1. Let $n \in \mathbb{N}$. We introduce the following initial-boundary value problem

$$
\left\{\begin{array}{l}
\partial_{t} u_{n}-\operatorname{div} A_{n}\left(x, t, u_{n}, \nabla u_{n}\right)=f \quad \text { in } \Omega_{T}, \\
u_{n}=0 \quad \text { on } \partial \Omega \times(0, T), \\
u_{n}(\cdot, 0)=u_{0} \quad \text { in } \Omega,
\end{array}\right.
$$

setting for $(x, t) \in \Omega_{T}$

$$
\theta_{n}(x, t):=\frac{\mathcal{T}_{n} \max \{b(x, t), \tilde{b}(x, t)\}}{\max \{b(x, t), \tilde{b}(x, t)\}} \quad \text { for a.e. }(x, t) \in \Omega_{T}
$$

(where we mean $\theta_{n}(x, t)=1$ if $b(x, t)=\tilde{b}(x, t)=0$ ) and

$$
A_{n}:(x, t, u, \xi) \in \Omega_{T} \times \mathbb{R} \times \mathbb{R}^{N} \mapsto A\left(x, t, \theta_{n} u, \xi\right) .
$$

The results of Sect. 4 provide the existence of a solution

$$
u_{n} \in C^{0}\left([0, T], L^{2}(\Omega)\right) \cap L^{p}\left(0, T, W_{0}^{1, p}(\Omega)\right)
$$

to problem (5.1). In fact, $A_{n}=A_{n}(x, t, u, \xi)$ satisfies (1.5), (1.4) with $\mathcal{T}_{n} b$ in place of $b$, and (1.6) with $\mathcal{T}_{n} \tilde{b}$ in place of $\tilde{b}$. To achieve the proof of Theorem 1.1 we need to pass to the limit in (5.1). Since $\mathcal{T}_{n} b \leqslant b$ in $\Omega_{T}$ for every $n \in \mathbb{N}$, by 
Proposition 3.2 with $\lambda=1$, there exists a positive constant independent of $n$ such that the following estimate for a solution to problem (5.1) holds

$$
\sup _{0<t<T} \int_{\Omega}\left|u_{n}(\cdot, t)\right|^{2} \mathrm{~d} x+\int_{\Omega_{T}}\left|\nabla u_{n}\right|^{p} \mathrm{~d} x \mathrm{~d} t \leqslant C
$$

Hence, there exists $u \in L^{\infty}\left(0, T, L^{2}(\Omega)\right) \cap L^{p}\left(0, T, W_{0}^{1, p}(\Omega)\right)$ such that (for a subsequence not relabeled)

$$
\begin{gathered}
u_{n} \rightarrow u \quad \text { weakly in } L^{p}\left(\Omega_{T}\right) \\
\nabla u_{n} \rightarrow \nabla u \quad \text { weakly in } L^{p}\left(\Omega_{T}, \mathbb{R}^{N}\right) \\
u_{n} \rightarrow^{*} u \quad \text { weakly }^{*} \text { in } L^{\infty}\left(0, T ; L^{2}(\Omega)\right)
\end{gathered}
$$

as $n \rightarrow \infty$. With the aid of the equation in (5.1), we obtain a uniform bound for the norm of the time derivative of $u_{n}$ in $L^{p^{\prime}}\left(0, T, W^{-1, p^{\prime}}(\Omega)\right)$. Therefore, by Aubin-Lions lemma we have

$$
\begin{gathered}
u_{n} \rightarrow u \quad \text { strongly in } L^{p}\left(\Omega_{T}\right) \text { and a.e. in } \Omega_{T} \\
\partial_{t} u_{n} \rightarrow \partial_{t} u \quad \text { weakly in } L^{p^{\prime}}\left(0, T, W^{-1, p^{\prime}}(\Omega)\right)
\end{gathered}
$$

Note also that $u \in C^{0}\left([0, T], L^{2}(\Omega)\right)$ and $u(\cdot, 0)=u_{0}$. As before, this is a consequence of Lemma 2.2 , the boundedness of $\left\{u_{n}\right\}_{n \in \mathbb{N}}$ in $W_{p}(0, T)$ and of the weak convergence $u_{n} \rightarrow u$ in $L^{2}(\Omega)$ for all $t \in[0, T]$.

In the last stage of our proof we want to pass to the limit in (5.1), showing that actually $u$ solves (1.1). For $z \in \mathbb{R}$, we set

$$
\gamma(z):=\int_{0}^{z} \frac{\mathrm{d} \zeta}{1+|\zeta|^{p}}
$$

Obviously, $\gamma \in C^{1}(\mathbb{R}), \gamma$ is odd, $|\gamma(z)| \leqslant|z|$ and $0 \leqslant \gamma^{\prime}(z) \leqslant 1$ for all $z \in$ $\mathbb{R}$. In particular, $\gamma$ is Lipschitz continuous in the whole of $\mathbb{R}$ and therefore $\gamma\left(u_{n}-u\right) \in L^{p}\left(0, T, W_{0}^{1, p}(\Omega)\right)$. Moreover, since $\gamma(0)=0$ we deduce from (5.4) and (5.6)

$$
\begin{array}{ll}
\gamma\left(u_{n}-u\right) \rightarrow 0 & \text { weakly in } L^{p}\left(0, T, W_{0}^{1, p}(\Omega)\right) \\
\gamma\left(u_{n}-u\right) \rightarrow 0 & \text { strongly in } L^{p}\left(\Omega_{T}\right) \text { and a.e. in } \Omega_{T}
\end{array}
$$

We observe that $\left.\gamma\left(u_{n}-u\right)\right|_{t=0}=0$. Testing equation (5.1) by $\gamma\left(u_{n}-u\right)$ we get

$$
\begin{gathered}
\left.\int_{\Omega} \Gamma\left(u_{n}-u\right)\right|_{t=T} \mathrm{~d} x+\int_{\Omega_{T}} A_{n}\left(x, t, u_{n}, \nabla u_{n}\right) \cdot \nabla \gamma\left(u_{n}-u\right) \mathrm{d} x \mathrm{~d} t \\
=-\int_{0}^{T}\left\langle\partial_{t} u, \gamma\left(u_{n}-u\right)\right\rangle \mathrm{d} t+\int_{0}^{T}\left\langle f, \gamma\left(u_{n}-u\right)\right\rangle \mathrm{d} t
\end{gathered}
$$

where $\Gamma(z):=\int_{0}^{z} \gamma(\zeta) \mathrm{d} \zeta$ for $z \in \mathbb{R}$. Moreover, we have $\nabla \gamma\left(u_{n}-u\right)=\gamma^{\prime}\left(u_{n}-\right.$ $u)\left(\nabla u_{n}-\nabla u\right)$. From (5.8) it follows that

$$
\lim _{n \rightarrow \infty} \int_{0}^{T}\left\langle\partial_{t} u, \gamma\left(u_{n}-u\right)\right\rangle \mathrm{d} t=0
$$




$$
\lim _{n \rightarrow \infty} \int_{0}^{T}\left\langle f, \gamma\left(u_{n}-u\right)\right\rangle \mathrm{d} t=0
$$

Then, as $\Gamma \geqslant 0$, by (5.10) we have

$$
\limsup _{n \rightarrow \infty} \int_{\Omega_{T}} A_{n}\left(x, t, u_{n}, \nabla u_{n}\right) \cdot \nabla \gamma\left(u_{n}-u\right) \mathrm{d} x \mathrm{~d} t \leqslant 0 .
$$

We claim that

$$
\lim _{n \rightarrow \infty} \int_{\Omega_{T}} A_{n}\left(x, t, u_{n}, \nabla u\right) \cdot \nabla \gamma\left(u_{n}-u\right) \mathrm{d} x \mathrm{~d} t=0 .
$$

In view of (5.4), to get (5.14) it suffices to show that

$\gamma^{\prime}\left(u_{n}-u\right) A_{n}\left(x, t, u_{n}, \nabla u\right)=\frac{A_{n}\left(x, t, u_{n}, \nabla u\right)}{1+\left|u_{n}-u\right|^{p}} \quad$ is compact in $L^{p^{\prime}}\left(\Omega_{T}, \mathbb{R}^{N}\right)$.

Preliminarily, we observe that combining (5.6) with the property that $\theta_{n} \rightarrow 1$ as $n \rightarrow \infty$, we have

$$
\frac{A_{n}\left(x, t, u_{n}, \nabla u\right)}{1+\left|u_{n}-u\right|^{p}} \rightarrow A(x, t, u, \nabla u) \quad \text { a.e. in } \Omega .
$$

On the other hand, we see that

$$
\left|\frac{A_{n}\left(x, t, u_{n}, \nabla u\right)}{1+\left|u_{n}-u\right|^{p}}\right|^{p^{\prime}} \leqslant C(\beta, p)\left[|\nabla u|^{p}+K^{p^{\prime}}+(\tilde{b}|u|)^{p}+\tilde{b}^{p}\right]
$$

Now, from the monotonicity condition (1.5), (5.13) and (5.14) we get

$$
\lim _{n \rightarrow \infty} \int_{\Omega_{T}}\left[A_{n}\left(x, t, u_{n}, \nabla u_{n}\right)-A_{n}\left(x, t, u_{n}, \nabla u\right)\right] \nabla \gamma\left(u_{n}-u\right) \mathrm{d} x \mathrm{~d} t=0
$$

As the integrand is nonnegative, we have (for a subsequence)

$$
\left[A_{n}\left(x, t, u_{n}, \nabla u_{n}\right)-A_{n}\left(x, t, u_{n}, \nabla u\right)\right] \nabla \gamma\left(u_{n}-u\right) \rightarrow 0 \quad \text { a.e. in } \Omega_{T} .
$$

Moreover, since $\gamma^{\prime}\left(u_{n}-u\right) \rightarrow 1$ a.e. in $\Omega_{T}$, the above in turn implies

$$
\left[A_{n}\left(x, t, u_{n}, \nabla u_{n}\right)-A_{n}\left(x, t, u_{n}, \nabla u\right)\right]\left(\nabla u_{n}-\nabla u\right) \rightarrow 0 \quad \text { a.e. in } \Omega_{T} .
$$

Arguing as in the proof of [17, Lemma 3.3], we see that

$$
\nabla u_{n} \rightarrow \nabla u \quad \text { a.e. in } \Omega_{T}
$$

and

$$
A_{n}\left(x, t, u_{n}, \nabla u_{n}\right) \rightarrow A(x, t, u, \nabla u) \quad \text { in } L^{p^{\prime}}\left(\Omega_{T}, \mathbb{R}^{N}\right) \text { weakly }
$$

and we conclude that $u$ solves the original problem (1.1).

Remark 5.1. For the equation (1.9), conditions (1.4) and condition (1.10) can be rephrased in terms of $\|h\|_{L^{\infty}(0, T)}$ and $\mu$. For such equation we have

$$
A(x, t, u, \xi)=\mu|\xi|^{p-2} \xi+|u|^{p-2} u\left[h(t) \frac{x}{|x|^{p}}+B(x, t)\right]
$$


By Young inequality it follows that

$A(x, t, u, \xi) \cdot \xi \geqslant\left(1-\frac{1}{p}\right) \mu|\xi|^{p}-\left(1-\frac{1}{p}\right) \mu^{-\frac{1}{p-1}}\left[\frac{|h(t)|}{|x|^{p-1}}+|B(x, t)|\right]^{\frac{p}{p-1}}|u|^{p}$

So condition (1.4) holds with $\alpha=\left(1-\frac{1}{p}\right) \mu$ and

$$
b(x, t)=\left(1-\frac{1}{p}\right)^{\frac{1}{p}} \mu^{-\frac{1}{p(p-1)}}\left[\frac{|h(t)|}{|x|^{p-1}}+|B(x, t)|\right]^{\frac{1}{p-1}}
$$

Using formula (2.10) and the fact that $|B| \in L^{\infty}\left(\Omega_{T}\right)$ we have

$$
\mathscr{D}_{b}=\omega_{N}^{\frac{1}{N}}\left(1-\frac{1}{p}\right)^{\frac{1}{p}} \mu^{-\frac{1}{p(p-1)}}\|h\|_{L^{\infty}(0, T)}^{\frac{1}{p-1}}
$$

Taking into account the explicit values of $\alpha$ and $S_{N, p}$, we see that (1.10) reduces to

$$
\|h\|_{L^{\infty}(0, T)}<\mu\left(\frac{N-p}{p}\right)^{p-1}
$$

\section{Asymptotic behavior}

This section is devoted to studying the time behavior of a solution to problem (1.1). Through this section we assume that (1.2)-(1.4), (1.6) are in charge and that condition (1.10) is satisfied.

For convenience we will denote by

$$
g(t):=\|f(t)\|_{W^{-1, p^{\prime}}(\Omega)}^{p^{\prime}}+\|H(t)\|_{L^{1}(\Omega)}+\|b(t)\|_{L^{p}(\Omega)}^{p}
$$

for a.e. $t \in[0, T]$. The first result of the present section is the following

Theorem 6.1. Under the above assumptions, any solution $u$ to problem (1.1) satisfies for all $t \in[0, T]$ the estimate

$$
\|u(t)\|_{L^{2}(\Omega)}^{2} \leqslant x(t)
$$

where $x(t)$ is the unique solution of the problem

$$
\left\{\begin{array}{l}
\dot{x}(t)=-M_{1}(x(t))^{p / 2}+C_{0} g(t) \\
x(0)=\left\|u_{0}\right\|_{L^{2}(\Omega)}^{2}
\end{array} \quad \text { if } p>2\right.
$$

or

$$
\left\{\begin{array}{l}
\dot{x}(t)=-M_{2} x(t)+C_{0} g(t) \\
x(0)=\left\|u_{0}\right\|_{L^{2}(\Omega)}^{2}
\end{array} \quad \text { if } p \leqslant 2\right.
$$

for some positive constants $C_{0}, M_{1}$ and $M_{2}$ which depend only on the data as follows

$$
M_{1}=M_{1}\left(N, p, \alpha,|\Omega|, \mathscr{D}_{b}\right)
$$




$$
\begin{gathered}
M_{2}=M_{2}\left(N, p, \alpha,|\Omega|, \mathscr{D}_{b},\|b\|_{L^{p}\left(\Omega_{T}\right)},\left\|u_{0}\right\|_{L^{2}(\Omega)},\|H\|_{L^{1}\left(\Omega_{T}\right)},\|f\|_{L^{p^{\prime}}\left(0, T, W^{-1, p^{\prime}}(\Omega)\right)}\right) \\
C_{0}=C_{0}\left(N, p, \alpha, \mathscr{D}_{b},\|b\|_{L^{p}\left(\Omega_{T}\right)},\left\|u_{0}\right\|_{L^{2}(\Omega)},\|H\|_{L^{1}\left(\Omega_{T}\right)},\|f\|_{L^{p^{\prime}}\left(0, T, W^{-1, p^{\prime}}(\Omega)\right)}\right)
\end{gathered}
$$

Proof. We test the equation in $(1.1)$ by $u \chi_{(0, t)}$ for $t \in(0, T)$ fixed. We get the following energy equality

$$
\frac{1}{2}\|u(t)\|_{L^{2}(\Omega)}^{2}+\int_{\Omega_{t}} A(x, s, u, \nabla u) \cdot \nabla u \mathrm{~d} x \mathrm{~d} s=\frac{1}{2}\left\|u_{0}\right\|_{L^{2}(\Omega)}^{2}+\int_{0}^{t}\langle f, u\rangle \mathrm{d} s
$$

We write down such equality first for $t=t_{1}$ and subsequently for $t=t_{2}>t_{1}$, we subtract the relations obtained in this way, we deduce

$$
\frac{1}{2}\left\|u\left(t_{2}\right)\right\|_{L^{2}(\Omega)}^{2}-\frac{1}{2}\left\|u\left(t_{1}\right)\right\|_{L^{2}(\Omega)}^{2}+\int_{t_{1}}^{t_{2}} \int_{\Omega} A(x, s, u, \nabla u) \cdot \nabla u \mathrm{~d} x \mathrm{~d} s=\int_{t_{1}}^{t_{2}}\langle f, u\rangle \mathrm{d} s
$$

Using (1.4) we get

$$
\begin{aligned}
\frac{1}{2}\left\|u\left(t_{2}\right)\right\|_{L^{2}(\Omega)}^{2} & -\frac{1}{2}\left\|u\left(t_{1}\right)\right\|_{L^{2}(\Omega)}^{2}+\alpha \int_{t_{1}}^{t_{2}} \int_{\Omega}|\nabla u|^{p} \mathrm{~d} x \mathrm{~d} s \\
& \leqslant \int_{t_{1}}^{t_{2}} \int_{\Omega}(b(x, s)|u|)^{p} \mathrm{~d} x \mathrm{~d} s+\int_{t_{1}}^{t_{2}} \int_{\Omega} H(x, s) \mathrm{d} x \mathrm{~d} s+\int_{t_{1}}^{t_{2}}\langle f, u\rangle \mathrm{d} s
\end{aligned}
$$

By Young inequality the latter inequality implies

$$
\begin{aligned}
& \frac{1}{2}\left\|u\left(t_{2}\right)\right\|_{L^{2}(\Omega)}^{2}-\frac{1}{2}\left\|u\left(t_{1}\right)\right\|_{L^{2}(\Omega)}^{2}+\alpha\|\nabla u\|_{L^{p}\left(\Omega \times\left(t_{1}, t_{2}\right)\right)}^{p} \\
& \leqslant\|b|u|\|_{L^{p}\left(\Omega \times\left(t_{1}, t_{2}\right)\right)}^{p}+\|H\|_{L^{1}\left(\Omega \times\left(t_{1}, t_{2}\right)\right)}+C(\varepsilon, p)\|f\|_{L^{p^{\prime}}\left(t_{1}, t_{2}, W^{-1, p^{\prime}}(\Omega)\right)}^{p^{\prime}} \\
& \quad+\varepsilon\|\nabla u\|_{L^{p}\left(\Omega \times\left(t_{1}, t_{2}\right)\right)}^{p}
\end{aligned}
$$

Taken $k, m>0$, we argue as in the proof of formula (3.17) and obtain

$$
\begin{aligned}
\|b|u|\|_{L^{p}\left(\Omega \times\left(t_{1}, t_{2}\right)\right)}^{p} \leqslant & {\left[S_{N, p}^{p} m^{p}\left(M_{0} \Psi(k)\right)^{p / N}\right.} \\
& \left.+S_{N, p}^{p}\left\|b \chi_{\{b>m\}}\right\|_{L^{\infty}\left(0, T, L^{N, \infty}(\Omega)\right)}^{p}\right]\|\nabla u\|_{L^{p}\left(\Omega \times\left(t_{1}, t_{2}\right)\right)}^{p} \\
& +k^{p}\|b\|_{L^{p}\left(\Omega \times\left(t_{1}, t_{2}\right)\right)}^{p}
\end{aligned}
$$

where $M_{0}$ is the constant appearing in Lemma 3.1. Suitable choices first of $m$ (we need that (3.19) holds true again) and subsequently of $k$ and of $\varepsilon$ give us

$$
\begin{aligned}
& \left\|u\left(t_{2}\right)\right\|_{L^{2}(\Omega)}^{2}-\left\|u\left(t_{1}\right)\right\|_{L^{2}(\Omega)}^{2}+C_{1}\|\nabla u\|_{L^{p}\left(\Omega \times\left(t_{1}, t_{2}\right)\right)}^{p} \\
& \quad \leqslant C_{0}\left(\|b\|_{L^{p}\left(\Omega \times\left(t_{1}, t_{2}\right)\right)}^{p}+\|H\|_{L^{1}\left(\Omega \times\left(t_{1}, t_{2}\right)\right)}+\|f\|_{L^{p^{\prime}\left(t_{1}, t_{2} \cdot W^{-1, p^{\prime}}(\Omega)\right)}}^{p^{\prime}}\right)
\end{aligned}
$$

where $C_{0}$ is a constant which depends only on $N, p, \alpha,\|b\|_{L^{p}\left(\Omega_{T}\right)}, \mathscr{D}_{b},\left\|u_{0}\right\|_{L^{2}(\Omega)}$, $\|H\|_{L^{1}\left(\Omega_{T}\right)},\|f\|_{L^{p^{\prime}}\left(0, T, W^{-1, p^{\prime}}(\Omega)\right)}$ and $C_{1}$ is a constant which depends only on $N, p, \alpha$ and $\mathscr{D}_{b}$. By (6.1) the latter relation may be rewritten as

$$
\left\|u\left(t_{2}\right)\right\|_{L^{2}(\Omega)}^{2}-\left\|u\left(t_{1}\right)\right\|_{L^{2}(\Omega)}^{2}+C_{1} \int_{t_{1}}^{t_{2}} \int_{\Omega}|\nabla u|^{p} \mathrm{~d} x \mathrm{~d} s \leqslant C_{0} \int_{t_{1}}^{t_{2}} g(s) \mathrm{d} s
$$


By Sobolev inequality we have

$$
\left\|u\left(t_{2}\right)\right\|_{L^{2}(\Omega)}^{2}-\left\|u\left(t_{1}\right)\right\|_{L^{2}(\Omega)}^{2}+C_{1} \hat{S}_{N, p}^{p} \int_{t_{1}}^{t_{2}}\|u\|_{L^{p^{*}}(\Omega)}^{p} \mathrm{~d} x \mathrm{~d} s \leqslant C_{0} \int_{t_{1}}^{t_{2}} g(s) \mathrm{d} s
$$

where $\hat{S}_{N, p}$ is the sharp constant for the classical Sobolev inequality

$$
\hat{S}_{N, p}\|h\|_{L^{p^{*}}(\Omega)} \leqslant\|\nabla h\|_{L^{p}(\Omega)} \quad \text { for every } h \in W_{0}^{1, p}(\Omega)
$$

Since $p>2 N /(N+2)$ is equivalent to $p^{*}>2$, then

$$
\left(f_{\Omega}|u(\cdot, s)|^{2} \mathrm{~d} x\right)^{p^{*} / 2} \leqslant f_{\Omega}|u(\cdot, s)|^{p^{*}} \mathrm{~d} x
$$

for a.e. $s \in\left(t_{1}, t_{2}\right)$. Hence, from (6.11)

$$
\left\|u\left(t_{2}\right)\right\|_{L^{2}(\Omega)}^{2}-\left\|u\left(t_{1}\right)\right\|_{L^{2}(\Omega)}^{2}+C_{1} \hat{S}_{N, p}^{p}|\Omega|^{\frac{p}{2}+\frac{p}{N}-1} \int_{t_{1}}^{t_{2}}\|u(s)\|_{L^{2}(\Omega)}^{p} \mathrm{~d} s \leqslant C_{0} \int_{t_{1}}^{t_{2}} g(s) \mathrm{d} s
$$

We distinguish two cases.

Case 1. $p>2$. In this case, the claimed result follows directly by Lemma 2.5 with $t_{0}=0, \gamma(t):=\|u(t)\|_{L^{2}(\Omega)}^{2}, \psi(t, y) \equiv \psi(y):=C_{1} \hat{S}_{N, p}^{p}|\Omega|^{\frac{p}{2}+\frac{p}{N}-1}$ $|y|^{p / 2}$.

Case 2. $2 N /(N+2)<p \leqslant 2$. By $(6.10)$ we have

$$
\|u(t)\|_{L^{2}(\Omega)}^{2} \leqslant \Lambda_{0}:=\left(\left\|u_{0}\right\|_{L^{2}(\Omega)}^{2}+C_{0} \int_{0}^{T} g(s) \mathrm{d} s\right)
$$

In turn, we have

$$
\|u(t)\|_{L^{2}(\Omega)}^{p}=\frac{\|u(t)\|_{L^{2}(\Omega)}^{2}}{\|u(t)\|_{L^{2}(\Omega)}^{2-p}} \geqslant \frac{\|u(t)\|_{L^{2}(\Omega)}^{2}}{\Lambda_{0}^{\frac{2-p}{2}}}
$$

and therefore from $(6.12)$

$$
\begin{aligned}
\left\|u\left(t_{2}\right)\right\|_{L^{2}(\Omega)}^{2} & -\left\|u\left(t_{1}\right)\right\|_{L^{2}(\Omega)}^{2}+C_{1} \hat{S}_{N, p}^{p}|\Omega|^{\frac{p}{2}+\frac{p}{N}-1} \Lambda_{0}^{\frac{p-2}{2}} \int_{t_{1}}^{t_{2}}\|u(s)\|_{L^{2}(\Omega)}^{2} \mathrm{~d} s \\
& \leqslant C_{0} \int_{t_{1}}^{t_{2}} g(s) \mathrm{d} s
\end{aligned}
$$

In this case, the claimed result follows again by Lemma 2.5 where we choose $t_{0}=0, \gamma(t):=\|u(t)\|_{L^{2}(\Omega)}^{2}, \psi(t, y) \equiv \psi(y):=C_{1} \hat{S}_{N, p}^{p}|\Omega|^{\frac{p}{2}+\frac{p}{N}-1} \Lambda_{0}^{\frac{p-2}{2}}|y|$.

As already observed, the constant $C_{0}$ depends only on $N, p, \alpha,\|b\|_{L^{p}\left(\Omega_{T}\right)}$, $\mathscr{D}_{b},\left\|u_{0}\right\|_{L^{2}(\Omega)},\|H\|_{L^{1}\left(\Omega_{T}\right)},\|f\|_{L^{p^{\prime}}\left(0, T, W^{-1, p^{\prime}}(\Omega)\right)}$. On the other hand, in (6.3) one may choose $M_{1}:=C_{1} \hat{S}_{N, p}^{p}|\Omega|^{\frac{p}{2}+\frac{p}{N}-1}$ and such a constant depends only on $N, p, \alpha,|\Omega|$ and $\mathscr{D}_{b}$ (since $C_{1}$ depends on $N, p, \alpha$, and $\mathscr{D}_{b}$ ). Similarly, in (6.4) one may choose $M_{2}:=C_{1} \hat{S}_{N, p}^{p}|\Omega|^{\frac{p}{2}+\frac{p}{N}-1} \Lambda_{0}^{\frac{p-2}{2}}$ and such a constant depends 
only on $N, p, \alpha,|\Omega|,\|b\|_{L^{p}\left(\Omega_{T}\right)}, \mathscr{D}_{b},\left\|u_{0}\right\|_{L^{2}(\Omega)},\|H\|_{L^{1}\left(\Omega_{T}\right)},\|f\|_{L^{p^{\prime}}\left(0, T, W^{-1, p^{\prime}}(\Omega)\right)}$ since $C_{0}$ and $\Lambda_{0}$ depend only on on $N, p, \alpha,\|b\|_{L^{p}\left(\Omega_{T}\right)}, \mathscr{D}_{b},\left\|u_{0}\right\|_{L^{2}(\Omega)},\|H\|_{L^{1}\left(\Omega_{T}\right)}$, $\|f\|_{L^{p^{\prime}}\left(0, T, W^{-1, p^{\prime}}(\Omega)\right)}$.

As a byproduct of previous result, we are able to show that the $L^{2}(\Omega)-$ norm of any solution to problem (1.1) decays as an explicit negative power of the time variable and exponentially fast respectively in case $p>2$ and $2 N /(N+2)<p \leqslant 2$ by using Lemma 2.6.

Corollary 6.2. Under the assumptions of Theorem 6.1, if $u$ is a solution to problem (1.1), then for any $t \in[0, T]$

$\|u(t)\|_{L^{2}(\Omega)}^{2} \leqslant \frac{\left\|u_{0}\right\|_{L^{2}(\Omega)}^{2}}{\left[1+\left(\frac{p}{2}-1\right) M_{1}\left\|u_{0}\right\|_{L^{2}(\Omega)}^{p-2} t\right]^{\frac{2}{p-2}}}+C_{0} \int_{0}^{t} g(s) \mathrm{d} s \quad$ if $p>2$

$\|u(t)\|_{L^{2}(\Omega)}^{2} \leqslant\left\|u_{0}\right\|_{L^{2}(\Omega)}^{2} e^{-M_{2} t}+C_{0} \int_{0}^{t} e^{-M_{2}(t-s)} g(s) \mathrm{d} s \quad$ if $\frac{2 N}{N+2}<p \leqslant 2$

for some positive constants $C_{0}, M_{1}$ and $M_{2}$ whose data-dependence is exactly as in (6.5), (6.6), (6.7).

Proof. We set again $\gamma(t):=\|u(t)\|_{L^{2}(\Omega)}^{2}$. Then by Lemma 2.6 we get

$$
x(t) \leqslant y(t):=\frac{\gamma(0)}{\left[1+\nu M_{1} \gamma(0)^{\nu} t\right]^{1 / \nu}}+C_{0} \int_{0}^{t} g(s) \mathrm{d} s
$$

choosing $\nu=p / 2-1$. The estimate (6.16) follows by solving the Cauchy problem (6.4).

Now we are able to describe the asymptotic behavior of a solution. From now on, we assume that (1.4) and (1.6) hold true in $\Omega_{\infty}:=\Omega \times(0,+\infty)$ with $H \in L^{1}\left(\Omega_{\infty}\right), K \in L^{p^{\prime}}\left(\Omega_{\infty}\right), \tilde{b} \in L^{\infty}\left(0, \infty, L^{N, \infty}(\Omega)\right)$ and

$$
b \in L^{p}\left(\Omega_{\infty}\right) \cap L^{\infty}\left(0, \infty, L^{N, \infty}(\Omega)\right)
$$

with

$$
\mathscr{D}_{b}^{\infty}:=\operatorname{dist}_{L^{\infty}\left(0, \infty ; L^{N, \infty}(\Omega)\right)}\left(b, L^{\infty}\left(\Omega_{\infty}\right)\right)<\frac{\alpha^{1 / p}}{S_{N, p}}
$$

Moreover, assume that $u_{0} \in L^{2}(\Omega)$ and that $f \in L^{p^{\prime}}\left(0, \infty, W^{-1, p^{\prime}}(\Omega)\right)$. Following line by line the proofs of Lemma 3.1 and Proposition 3.2, we are able to prove the next result.

Proposition 6.3. Let $u \in C_{\mathrm{loc}}^{0}\left([0, \infty), L^{2}(\Omega)\right) \cap L_{\mathrm{loc}}^{p}\left(0, \infty, W_{0}^{1, p}(\Omega)\right)$ be a solution to problem (1.1) in $\Omega_{\infty}$. For every $k>0$ we have

$$
\sup _{0<t<\infty}|\{x \in \Omega:|u(x, t)|>k\}| \leqslant M_{0}^{\infty} \Psi(k)
$$


where

$$
M_{0}^{\infty}=\frac{1}{2}\left\|u_{0}\right\|_{L^{2}(\Omega)}^{2}+\|H\|_{L^{1}\left(\Omega_{\infty}\right)}+\|b\|_{L^{p}\left(\Omega_{\infty}\right)}^{p}+\alpha^{-1 /(p-1)}\|f\|_{L^{p^{\prime}}\left(0, \infty, W^{-1, p^{\prime}}(\Omega)\right)}^{p^{\prime}}
$$

Moreover, the following estimate holds

$$
\sup _{0<t<\infty} \int_{\Omega}|u(\cdot, t)|^{2} \mathrm{~d} x+\int_{\Omega_{\infty}}|\nabla u|^{p} \mathrm{~d} x \mathrm{~d} t \leqslant C
$$

for some positive constant $C$ depending only on $N, p, \alpha,\|b\|_{L^{p}\left(\Omega_{\infty}\right)}, \mathscr{D}_{b}^{\infty}$, $\left\|u_{0}\right\|_{L^{2}(\Omega)},\|H\|_{L^{1}\left(\Omega_{\infty}\right)},\|f\|_{L^{p^{\prime}}\left(0, \infty, W^{-1, p^{\prime}}(\Omega)\right)} \cdot$

As a consequence, we obtain the following result.

Proposition 6.4. Let $u \in C_{\mathrm{loc}}^{0}\left([0, \infty), L^{2}(\Omega)\right) \cap L_{\mathrm{loc}}^{p}\left(0, \infty, W_{0}^{1, p}(\Omega)\right)$ be a solution to problem (1.1) in $\Omega_{\infty}$. Then, for every $t \in(0, \infty)$

$$
\begin{aligned}
\|u(t)\|_{L^{2}(\Omega)}^{2} \leqslant & {\left[\left(\frac{p}{2}-1\right) M_{1}\right]^{-\frac{2}{p-2}} t^{-\frac{2}{p-2}}+C_{0} \int_{t / 2}^{t} g(s) \mathrm{d} s \quad \text { if } p>2 } \\
\|u(t)\|_{L^{2}(\Omega)}^{2} \leqslant & \left(\left\|u_{0}\right\|_{L^{2}(\Omega)}^{2}+C_{0}\|g\|_{L^{1}([0, \infty))}\right) e^{-\frac{M_{2}}{2} t} \\
& +C_{0} \int_{t / 2}^{t} e^{-\frac{M_{2}}{2}(t-s)} g(s) \mathrm{d} s \quad \text { if } \frac{2 N}{N+2}<p \leqslant 2
\end{aligned}
$$

for some positive constants $C_{0}, M_{1}$ and $M_{2}$ which depend only on the data as follows

$$
\begin{aligned}
& M_{1}=M_{1}\left(N, p, \alpha,|\Omega|, \mathscr{D}_{b}^{\infty}\right) \\
& M_{2}=M_{2}\left(N, p, \alpha,|\Omega|, \mathscr{D}_{b}^{\infty},\|b\|_{L^{p}\left(\Omega_{\infty}\right)},\left\|u_{0}\right\|_{L^{2}(\Omega)},\|H\|_{L^{1}\left(\Omega_{\infty}\right)},\|f\|_{L^{p^{\prime}}\left(0, \infty, W^{-1, p^{\prime}}(\Omega)\right)}\right) \\
& C_{0}=C_{0}\left(N, p, \alpha, \mathscr{D}_{b}^{\infty},\|b\|_{L^{p}\left(\Omega_{\infty}\right)},\left\|u_{0}\right\|_{L^{2}(\Omega)},\|H\|_{L^{1}\left(\Omega_{\infty}\right)},\|f\|_{L^{p^{\prime}}\left(0, \infty, W^{-1, p^{\prime}}(\Omega)\right)}\right)
\end{aligned}
$$

The proof follows the same lines as in Proposition 3.1 and Proposition 3.2 of $[18]$.

Remark 6.5. The statement of Theorem 6.1 improves the behavior in time of a solution to problem (1.1) known so far $[10,18]$ for $p$-Laplace operator. Related results in the case $p=2$ are available in $[4,7,8]$.

\section{Acknowledgements}

The Authors express their gratitude to the referee for all valuable comments helping to concretely improve exposition of the results.

Funding Open access funding provided by Università degli Studi di Napoli Federico II within the CRUI-CARE Agreement. 
Open Access. This article is licensed under a Creative Commons Attribution 4.0 International License, which permits use, sharing, adaptation, distribution and reproduction in any medium or format, as long as you give appropriate credit to the original author(s) and the source, provide a link to the Creative Commons licence, and indicate if changes were made. The images or other third party material in this article are included in the article's Creative Commons licence, unless indicated otherwise in a credit line to the material. If material is not included in the article's Creative Commons licence and your intended use is not permitted by statutory regulation or exceeds the permitted use, you will need to obtain permission directly from the copyright holder. To view a copy of this licence, visit http://creativecommons. org/licenses/by/4.0/.

Publisher's Note Springer Nature remains neutral with regard to jurisdictional claims in published maps and institutional affiliations.

\section{References}

[1] Alvino, A.: Sulla disuguaglianza di Sobolev in Spazi di Lorentz, Boll. Un. Mat. It. A $(5) 14,148-156(1977)$

[2] Bennett, C., Sharpley, R.: Interpolation of Operators. Academic Press, London (1988)

[3] Boccardo, L., Orsina, L., Porretta, A.: Some noncoercive parabolic equations with lower order terms in divergence form. J. Evol. Equ. 3, 407-418 (2003)

[4] Boccardo, L., Orsina, L., Porzio, M.M.: Regularity results and asymptotic bahavior for a noncoercive parabolic problem. J. Evol. Equ. Published online, (2021)

[5] Cardaliaguet, P., Lasry, J.-M., Lions, P.-L., Porretta, A.: Long time average of mean field games. Netw. Heterog. Media 7(2), 279-301 (2012)

[6] Fang, W., Ito, K.: Weak solutions for diffusion-convection equations. Appl. Math. Lett. 13(3), 69-75 (2000)

[7] Farroni, F.: Asymptotic stability estimates for some evolution problems with singular convection field. Ric. Mat. (2020). https://doi.org/10.1007/ s11587-020-00537-1

[8] Farroni, F., Moscariello, G.: A nonlinear parabolic equation with drift term, Part B. Nonlinear Anal 177, 397-412 (2018)

[9] Farroni, F., Greco, L., Moscariello, G., Zecca, G.: Noncoercive quasilinear elliptic operators with singular lower order terms. Calc. Var. and Partial Differential Equations 60, 83 (2021). https://doi.org/10.1007/s00526-021-1965-z

[10] Frankowska, H., Moscariello, G.: Long-Time behavior of solutions to an evolution PDE with nonstandard growth. Adv. Calc. Var. (2020). https://doi.org/10. 1515/acv-2019-0061

[11] Gilbarg, D., Trudinger, N.S.: Elliptic Partial Differential Equations of Second Order. Springer, Berlin (1983) 
[12] Giannetti, F., Greco, L., Moscariello, G.: Linear elliptic equations with lowerorder terms. Differ. Integr. Equ. 26(5-6), 623-638 (2013)

[13] Greco, L., Moscariello, G., Zecca, G.: An obstacle problem for noncoercive operators, Abstract and Applied Analysis, Article ID 890289, Volume 2015, Article ID 8902898 pag. https://doi.org/10.1155/2015/890289

[14] Herrero, M.A., Vázquez, J.L., Asymptotic behaviour of the solutions of a strongly nonlinear parabolic problem. Ann. Fac. Sci. Toulouse Math. (5)3, no. 2, 113-127 (1981)

[15] Ladyženskaja, O. , Solonnikov, V.A. , Ural'ceva, N.N., Linear and quasilinear equations of parabolic type, Translations of the American Mathematical Society, American Mathematical Society, Providence, (1968)

[16] Lions, J.L.: Quelques méthodes de résolution des problèmes aux limites non linéaires. Dunod et Gauthier-Villars, Paris (1969)

[17] Leray, J., Lions, J.L.: Quelques résultats de Visik sur le problèmes elliptiques non linéaires par les méthodes de Minty-Browder. Bull. Soc. Math. France 93, 97-107 (1965)

[18] Moscariello, G., Porzio, M.M.: Quantitative asymptotic estimates for evolution problems. Nonlinear Anal. 154, 225-240 (2017)

[19] Moscariello, G., Porzio, M.M.: On the behavior in time of solutions to motion of non-Newtonian fluids. Nonlinear Differ. Equ. Appl. 27, 42 (2020). https://doi. org/10.1007/s00030-020-00645-9

[20] O'Neil, R.: Convolutions operators and $L(p, q)$ spaces. Duke Math. J. 30, 129$142(1963)$

[21] Porretta, A.: Weak solutions to Fokker-Planck equations and mean field games. Arch. Ration. Mech. Anal. 216(1), 1-62 (2015)

[22] Porzio, M.M., Asymptotic behavior and regularity properties of strongly nonlinear parabolic equations. Ann. Mat. Pura Appl. (4) 198(5), 1803-1833 (2019)

[23] Showalter, R.E., Monotone operators in Banach space and nonlinear partial differential equations. Mathematical Surveys and Monographs, 49. American Mathematical Society, Providence, RI, (1997). xiv+278 pp. ISBN: 0-8218-05002

Fernando Farroni, Gioconda Moscariello and Gabriella Zecca

Dipartimento di Matematica e Applicazioni R. Caccioppoli

Università degli Studi di Napoli Federico II

Complesso Monte S. Angelo, via Cinthia

80126 Naples

Italy

e-mail: gmoscari@unina.it

Fernando Farroni

e-mail: fernando.farroni@unina.it 
Gabriella Zecca

e-mail: g.zecca@unina.it

\section{Luigi Greco}

Dipartimento di Ingegneria Elettrica e delle Tecnologie dell'Informazione Università degli Studi di Napoli Federico II

Piazzale Tecchio, 80

80126 Naples

Italy

e-mail: luigreco@unina.it

Received: 28 December 2020.

Accepted: 20 April 2021. 